\title{
POINTWISE BOUNDS ON QUASIMODES OF SEMICLASSICAL SCHRÖDINGER OPERATORS IN DIMENSION TWO
}

\author{
Hart F. Smith ANd Maciej Zworski
}

\begin{abstract}
We prove sharp pointwise bounds on quasimodes of semiclassical Schrödinger operators with arbitrary smooth real potentials in dimension two. This end-point estimate was left open in the general study of semiclassical $L^{p}$ bounds conducted by Koch et al. [2]. However, we show that the results of [2] imply the two-dimensional end-point estimate by scaling and localization.
\end{abstract}

\section{Introduction}

Let $\mathrm{g}_{i j}(x)$ be a positive-definite Riemannian metric on $\mathbb{R}^{2}$ with the corresponding Laplace-Beltrami operator,

$$
\Delta_{\mathrm{g}} u:=\frac{1}{\sqrt{\mathrm{g}}} \sum_{i, j} \partial_{x_{j}}\left(\mathrm{~g}^{i j} \sqrt{\overline{\mathrm{g}}} \partial_{x_{j}} u\right), \quad\left(\mathrm{g}^{i j}\right):=\left(\mathrm{g}_{i j}\right)^{-1}, \quad \overline{\mathrm{g}}:=\operatorname{det}\left(\mathrm{g}_{i j}\right),
$$

and let $V \in C^{\infty}\left(\mathbb{R}^{2}\right)$ be real valued. We prove the following general bound, the analogue of which was already established (under an additional necessary condition) in higher dimensions in [2], but which was open in dimension two:

Theorem 1. Suppose that $h \leq 1$, and $u \in H_{\mathrm{loc}}^{2}\left(\mathbb{R}^{2}\right)$. Suppose that $u$ satisfies

$$
\left\|-h^{2} \Delta_{\mathrm{g}} u+V u\right\|_{L^{2}} \leq h, \quad\|u\|_{L^{2}} \leq 1
$$

Then for all $K \Subset \mathbb{R}^{2}$,

$$
\sup _{x \in K}|u(x)| \leq C_{K} h^{-\frac{1}{2}}
$$

where the constant $C_{K}$ depends only on $\mathrm{g}, V$, and $K$.

A function $u$ satisfying (1.1) is sometimes called a weak quasimode. It is a local object in the sense that if $u$ is a weak quasimode then $\psi u, \psi \in C_{c}^{\infty}\left(\mathbb{R}^{2}\right)$ is also one, so the theorem is easily reformulated with $g, V$, and $u$ defined on an open subset of $\mathbb{R}^{2}$. The localization is also valid in phase space: for instance if $\chi \in C_{c}^{\infty}\left(\mathbb{R}^{2} \times \mathbb{R}^{2}\right)$ then $\chi^{w}(x, h D) u$ is also a weak quasimode - see [1, Chapter 7] or [4, Chapter 4] for the review of the Weyl quantization $\chi \mapsto \chi^{w}$.

Received by the editors September 16, 2012. 
If $\liminf _{|x| \rightarrow \infty} V>0$, then $-h^{2} \Delta+V$ (defined on $C_{c}^{\infty}\left(\mathbb{R}^{2}\right)$ ) is essentially selfadjoint and the spectrum of $-h^{2} \Delta+V$ is discrete in a neighborhood of $0-$ see for instance [1, Chapter 4]. In this case, weak quasimodes arise as spectral clusters:

$$
u=\sum_{\left|E_{j}\right| \leq C h} c_{j} w_{j}, \quad\left(-h^{2} \Delta+V\right) w_{j}=E_{j} w_{j}, \quad\left\langle w_{j}, w_{k}\right\rangle_{L^{2}}=\delta_{j k}, \quad \sum_{j}\left|c_{j}\right|^{2} \leq 1 .
$$

Then $u$ is a weak quasimode in the sense of (1.1). Since $V(x) \geq c_{0}>0$ for $|x| \geq R$, Agmon estimates (see for instance [1, Chapter 6]) and Sobolev embedding show that $|u(x)| \leq e^{-c_{1} / h}, c_{1}>0$, for $|x| \geq R$. Hence we get global bounds

$$
|u(x)| \leq C h^{-\frac{1}{2}}, \quad x \in \mathbb{R}^{2} .
$$

It should be stressed however that a weak quasimode is a more general notion than a spectral cluster.

The result also holds when $\mathbb{R}^{2}$ is replaced by a two-dimensional manifold and, as in the example above, gives global bounds on spectral clusters (1.3) when the manifold is compact. If $V<0$ this is also a by-product of of the Avakumovic-LevitanHörmander bound on the spectral function - see [3], and for a proof of a semiclassical generalization see [2, Section 3] or [4, Section 7.4].

In higher dimensions the theorem requires an additional phase space localization assumption and is a special case of [2, Theorem 6]: Suppose $p(x, \xi)$ is a function on $\mathbb{R}^{n} \times \mathbb{R}^{n}$ satisfying $\partial_{x}^{\alpha} \partial_{\xi}^{\beta} p(x, \xi)=\mathcal{O}\left(\langle\xi\rangle^{m}\right)$ for some $m$. Suppose that $K \Subset \mathbb{R}^{n}$ and $\chi \in C_{c}^{\infty}\left(\mathbb{R}^{n} \times \mathbb{R}^{n}\right)$, and that for $(x, \xi) \in \operatorname{supp} \chi$

$$
p(x, \xi)=0, \quad d_{\xi} p(x, \xi)=0 \Longrightarrow d_{\xi}^{2} p(x, \xi) \text { is non-degenerate. }
$$

Then for $u(h)$ such that

$$
\operatorname{supp} u(h) \subset K, \quad u(h)=\chi^{w}(x, h D) u+\mathcal{O}_{\mathscr{S}}\left(h^{\infty}\right),
$$

we have

$$
\|u(h)\|_{\infty} \leq C h^{-\frac{n-1}{2}}\left(\|u(h)\|_{L^{2}}+\frac{1}{h}\left\|p^{w}(x, h D) u\right\|_{L^{2}}\right), \quad n \geq 3 .
$$

When $n=2$ the bound holds with $(\log (1 / h) / h)^{\frac{1}{2}}$, which is optimal in general if $d_{\xi}^{2} p$ is not positive definite - see [2, Sections 3 and 6$]$ and Section 3 below for examples.

A small bonus for Schrödinger operators in dimension two is the fact that the frequency localization condition in (1.4) required for (1.5) is not necessary - see (2.5) below. Furthermore, as noted already, in all dimensions the compact support condition on $u$ is easily dropped when working with local estimates on $u$.

The proof of Theorem 1 is reduced to a local result presented in Proposition 3. That result follows in turn from a rescaling argument involving several cases, some of which use the following result that forms part of [2, Corollary 1]. 
Theorem 2. Suppose that $u=u(h)$ satisfies (1.1), and that (1.4) holds. If $V(x) \neq 0$ for $x \in \operatorname{supp} u$, or if $\mathrm{g}^{i j}$ is positive definite and $d V(x) \neq 0$ for $x \in \operatorname{supp} u$, then

$$
\|u\|_{L^{\infty}}=\mathcal{O}\left(h^{-\frac{n-1}{2}}\right), \quad n \geq 2 .
$$

This result is the basis for Propositions 4 and 5 used in our proof. The case of Theorem 2 with $d V \neq 0$ is the most technically involved result in the paper [2]. We do not know of any simpler way to obtain the bound (1.2).

\section{Proof of Theorem 1}

By compactness of $K$, it suffices to prove uniform $L^{\infty}$ bounds on $u$ over a small ball about each point in $K$, where in our case the diameter of the ball can be taken to depend only on $\mathcal{C}^{N}$ estimates for $\mathrm{g}$ and $V$ over a unit sized neighborhood of $K$, for some large $N$. Without loss of generality we consider a ball centered at the origin in $\mathbb{R}^{2}$. Let

$$
B=\left\{x \in \mathbb{R}^{2}:|x|<1\right\}, \quad B^{*}=\left\{x \in \mathbb{R}^{2}:|x|<2\right\} .
$$

After a linear change of coordinates, we may assume that

$$
\mathrm{g}^{i j}(0)=\delta^{i j}
$$

Next, by replacing $V(x)$ by $c V(c x)$ and $\mathrm{g}^{i j}(x)$ by $\mathrm{g}^{i j}(c x)$, for some constant $c \leq$ 1 depending on the $\mathcal{C}^{2}$ norm of $\mathrm{g}$ and $V$ over a unit neighborhood of $K$, we may assume that

$$
\sup _{x \in B^{*}}|V(x)|+|d V(x)| \leq 2, \quad \sup _{x \in B^{*}}\left|d^{2} V(x)\right|+\sum_{i, j=1}^{2}\left|d g^{i j}(x)\right| \leq .01 .
$$

This has the effect of multiplying $h$ by a constant in the equation (1.1), which can be absorbed into the constant $C_{K}$ in (1.2).

In general, we let

$$
C_{N}=\sup _{x \in B^{*}} \sup _{|\alpha| \leq N}\left(\left|\partial^{\alpha} V(x)\right|+\sum_{i, j=1}^{2}\left|\partial^{\alpha} g^{i j}(x)\right|\right),
$$

and will deduce Theorem 1 as a corollary of the following:

Proposition 3. Suppose $h \leq 1$, that $\mathrm{g}, V$ satisfy (2.1) and (2.2), and that $u$ satisfies

$$
\left\|-h^{2} \Delta_{\mathrm{g}} u+V u\right\|_{L^{2}\left(B^{*}\right)} \leq h, \quad\|u\|_{L^{2}\left(B^{*}\right)} \leq 1 .
$$

Then

$$
\|u\|_{L^{\infty}(B)} \leq C h^{-\frac{1}{2}}
$$

where the constant $C$ depends only on $C_{N}$ in (2.3) for some fixed $N$.

We start the proof of Proposition 3 by recording the following two propositions, which are consequences of Theorem 2 . 
Proposition 4. Suppose that both (2.1)-(2.2) hold, and that $\frac{1}{2} \leq|V(x)| \leq 2$ for $|x| \leq 2$. If the following holds, and $h \leq 1$,

$$
\left\|-h^{2} \Delta_{\mathrm{g}} u+V u\right\|_{L^{2}\left(B^{*}\right)} \leq h, \quad\|u\|_{L^{2}\left(B^{*}\right)} \leq 1,
$$

then $\|u\|_{L^{\infty}(B)} \leq C h^{-\frac{1}{2}}$, where $C$ depends only on $C_{N}$ in (2.3) for some fixed $N$.

Proposition 5. Suppose that both (2.1)-(2.2) hold, and that $V(0)=0$ and $|d V(0)|=$ 1. If the following holds, and $h \leq 1$,

$$
\left\|-h^{2} \Delta_{\mathrm{g}} u+V u\right\|_{L^{2}\left(B^{*}\right)} \leq h, \quad\|u\|_{L^{2}\left(B^{*}\right)} \leq 1,
$$

then $\|u\|_{L^{\infty}(B)} \leq C h^{-\frac{1}{2}}$, where $C$ depends only on $C_{N}$ in (2.3) for some fixed $N$.

To see that these follow from Theorem 2, we first may assume that $u$ is compactly supported in $|x|<\frac{3}{2}$. Indeed, the assumptions imply $\|d u\|_{L^{2}(|x|<3 / 2)} \lesssim h^{-1}$, so that one may cut off $u$ by a smooth function which is supported in $|x|<\frac{3}{2}$ and equals 1 for $|x|<1$ without affecting the hypotheses. We may then modify g and $V$ outside $B^{*}$ so that $(2.2)-(2.3)$ are global bounds.

In Proposition 5 above, since $\left|d^{2} V\right| \leq .01$, we have $.98 \leq|d V(x)| \leq 1.02$ for $|x| \leq 2$, so since $\mathrm{g}$ is positive definite the conditions on $\mathrm{g}$ and $V$ in Theorem 2 are met. We remark that the conditions of Proposition 5 guarantee that the zero set of $V$ is a nearly-flat curve through the origin, although this is not strictly needed to apply the results of [2]. That the resulting constant $C$ depends only on $C_{N}$ for some fixed finite $N$ follows from the proofs in [2].

Finally, the condition in (1.4) that $u-\chi^{w}(x, h D) u=\mathcal{O}_{\mathscr{S}}\left(h^{\infty}\right)$ for some $\chi \in \mathcal{C}_{c}^{\infty}$ is not needed for Theorem 2 to hold for positive definite $\mathrm{g}^{i j}$ in dimension two. To see this, we note that if $|V|<2$ and $\left|g^{i j}(x)-\delta_{i j}\right| \leq 0.02$ on the ball $|x|<2$, then if $u$ is supported in $|x|<\frac{3}{2}$ and $\varphi(\xi)=1$ for $|\xi|<4$, condition (1.1) implies that

$$
\left\|(h D)^{2}(u-\varphi(h D) u)\right\|_{L^{2}}=\mathcal{O}(h) .
$$

This follows by the semiclassical pseudodifferential calculus (see [4, Theorem 4.29]), since for $\varphi_{0} \in C_{c}^{\infty}\left(\mathbb{R}^{2}\right)$ with $\operatorname{supp} \varphi_{0} \subset B^{*}, \varphi_{0}(x)(1-\varphi(\xi))|\xi|^{2} /\left(|\xi|_{\mathrm{g}}^{2}+V(x)\right) \in$ $S\left(\mathbb{R}^{2} \times \mathbb{R}^{2}\right)$.

Hence, writing $\hat{u}(\xi)$ for the standard Fourier transform of $u$,

$$
\begin{aligned}
\|u-\varphi(h D) u\|_{L^{\infty}} & \leq \frac{1}{(2 \pi)^{2}} \int_{\mathbb{R}^{2}}|1-\varphi(h \xi) \| \hat{u}(\xi)| d \xi \\
& \leq C \int|h \xi|^{2}|1-\varphi(h \xi) \| \hat{u}(\xi)|\left(1+|h \xi|^{2}\right)^{-1} d \xi \\
& \leq C\left\|(h D)^{2}(u-\varphi(h D) u)\right\|_{L^{2}}\left(\int_{\mathbb{R}^{2}}\left(1+|h \xi|^{2}\right)^{-2} d \xi\right)^{\frac{1}{2}} \\
& \leq C h h^{-1}=C,
\end{aligned}
$$

an even better estimate than required. Hence, we are reduced to proving estimates on $\varphi(h D) u$, which by compact support of $u$ satisfies (1.4).

We supplement Propositions 4 and 5 with the following two lemmas. 
Lemma 6. Suppose that (2.1)-(2.2) hold, and that $|V(x)| \leq 99$ hor $|x| \leq 2 h^{\frac{1}{2}}$. If the following holds, and $h \leq 1$,

$$
\left\|-h^{2} \Delta_{\mathrm{g}} u+V u\right\|_{L^{2}\left(|x|<2 h^{1 / 2}\right)} \leq h, \quad\|u\|_{L^{2}\left(|x|<2 h^{1 / 2}\right)} \leq 1,
$$

then $\|u\|_{L^{\infty}\left(|x|<h^{1 / 2}\right)} \leq C h^{-\frac{1}{2}}$, where $C$ depends only on $C_{N}$ in (2.3) for some fixed $N$.

Proof. Consider the function $\tilde{u}(x)=h^{\frac{1}{2}} u\left(h^{\frac{1}{2}} x\right)$, and $\tilde{\mathrm{g}}^{i j}(x)=\mathrm{g}^{i j}\left(h^{\frac{1}{2}} x\right)$. Then, since $\|V u\|_{L^{2}\left(|x|<2 h^{1 / 2}\right)} \leq 99 h$, we have

$$
\left\|\Delta_{\tilde{\mathrm{g}}} \tilde{u}\right\|_{L^{2}(|x|<2)} \leq 100, \quad\|\tilde{u}\|_{L^{2}(|x|<2)} \leq 1
$$

Since the spatial dimension is 2 , interior Sobolev estimates yield $\|\tilde{u}\|_{L^{\infty}(|x|<1)} \leq C$, where we note that the conditions (2.1) and (2.2) hold for $\tilde{\mathrm{g}}$ since $h^{\frac{1}{2}} \leq 1$.

Lemma 7. Suppose that both (2.1)-(2.2) hold, and that $\frac{1}{2} c \leq|V(x)| \leq 2 c$ for $|x| \leq 2 c^{\frac{1}{2}}$. If the following holds, and $h \leq c \leq 1$,

$$
\left\|-h^{2} \Delta_{\mathrm{g}} u+V u\right\|_{L^{2}\left(|x|<2 c^{1 / 2}\right)} \leq h, \quad\|u\|_{L^{2}\left(|x|<2 c^{1 / 2}\right)} \leq 1,
$$

then $\|u\|_{L^{\infty}\left(|x|<c^{1 / 2}\right)} \leq C h^{-\frac{1}{2}}$, where $C$ depends only $C_{N}$ in (2.3) for some fixed $N$.

Proof. Let $\tilde{u}(x)=c^{\frac{1}{2}} u\left(c^{\frac{1}{2}} x\right), \tilde{\mathrm{g}}^{i j}(x)=\mathrm{g}^{i j}\left(c^{\frac{1}{2}} x\right)$, and $\tilde{V}(x)=c^{-1} V\left(c^{\frac{1}{2}} x\right)$. Note that the assumptions on $V(x)$ in the statement and in (2.2) imply that $|d V(x)| \leq c^{\frac{1}{2}}$ for $|x|<2 c^{1 / 2}$, so that $\tilde{V}$ satisfies $(2.2)$, and the constants $C_{N}$ in (2.3) can only decrease for $c \leq 1$. Then with $\tilde{h}=c^{-1} h \leq 1$,

$$
\left\|-\tilde{h}^{2} \Delta_{\tilde{\mathrm{g}}} \tilde{u}+\tilde{V} \tilde{u}\right\|_{L^{2}(|x|<2)} \leq \tilde{h}, \quad\|\tilde{u}\|_{L^{2}(|x|<2)} \leq 1 .
$$

By Proposition 4, we have $\|\tilde{u}\|_{L^{\infty}(|x|<1)} \leq C \tilde{h}^{-\frac{1}{2}}$, giving the desired result.

Proof of Proposition 3. It suffices to prove that for each $\left|x_{0}\right|<1$ there is some $\frac{1}{2} \geq r>0$, so that $\|u\|_{L^{\infty}\left(\left|x-x_{0}\right|<r\right)} \leq C h^{-\frac{1}{2}}$, with a global constant $C$. Without loss of generality we take $x_{0}=0$.

We will split consideration up into four cases, depending on the relative size of $|V(0)|$ and $|d V(0)|$. Since for $h$ bounded away from 0 the result follows by elliptic estimates, we will assume $h \leq \frac{1}{4}$ so that $h^{\frac{1}{2}}$ below is at most $\frac{1}{2}$.

Case 1: $|V(0)| \leq h,|d V(0)| \leq 8 h^{\frac{1}{2}}$. Since $\left|d^{2} V(x)\right| \leq 0.01$, then Lemma 6 applies to give the result with $r=h^{\frac{1}{2}}$.

Case 2: $|V(0)| \leq h,|d V(0)| \geq 8 h^{\frac{1}{2}}$. Since we may add a constant of size $h$ to $V$ without affecting $(2.4)$, we may assume $V(0)=0$. By rotating we may then assume

$$
V(x)=\beta x_{1}+f_{i j}(x) x_{i} x_{j}
$$


where $\beta=|d V(0)| \geq 8 h^{\frac{1}{2}}$. Dividing $V$ by 4 if necessary we may assume $\beta \leq \frac{1}{2}$. Let $\tilde{u}=\beta u(\beta x), \tilde{\mathrm{g}}^{i j}(x)=\mathrm{g}^{i j}(\beta x)$, and

$$
\tilde{V}(x)=\beta^{-2} V(\beta x)=x_{1}+f_{i j}(\beta x) x_{i} x_{j} .
$$

With $\tilde{h}=\beta^{-2} h<1$ we have

$$
\left\|-\tilde{h}^{2} \Delta_{\tilde{g}} \tilde{u}+\tilde{V} \tilde{u}\right\|_{L^{2}(|x|<2)} \leq \tilde{h}, \quad\|\tilde{u}\|_{L^{2}(|x|<2)} \leq 1 .
$$

Proposition 5 applies, since $\tilde{g}$ and $\tilde{V}$ satisfy (2.1)-(2.2), and the constants $C_{N}$ in (2.3) for $\tilde{g}$ and $\tilde{V}$ are bounded by those for $g$ and $V$. Thus, $\|\tilde{u}\|_{L^{\infty}(|x|<1)} \leq C \tilde{h}^{-\frac{1}{2}}$, giving the desired result on $u$ with $r=|d V(0)|$.

Case 3: $|V(0)| \geq h,|d V(0)| \leq 9|V(0)|^{\frac{1}{2}}$. In this case, with $c=|V(0)|$, it follows that $\frac{1}{2} c \leq|V(x)| \leq 2 c$ for $|x| \leq \frac{1}{20} c^{\frac{1}{2}}$. We may apply Lemma 7 with $V$ replaced by $\frac{1}{1600} V$ to get the desired result with $r=\frac{1}{40}|V(0)|^{\frac{1}{2}}$.

Case 4: $|V(0)| \geq h,|d V(0)| \geq 9|V(0)|^{\frac{1}{2}}$. Since $\left|d^{2} V(x)\right| \leq .01$, it follows that there is a point $x_{0}$ with $\left|x_{0}\right| \leq \frac{1}{8}|V(0)|^{\frac{1}{2}}$ where $V\left(x_{0}\right)=0$. Since $\left|d V\left(x_{0}\right)\right| \geq 8|V(0)|^{\frac{1}{2}} \geq 8 h^{\frac{1}{2}}$, we may translate and apply Case 2 to get $L^{\infty}$ bounds on $u$ over a neighborhood of radius $\left|d V\left(x_{0}\right)\right|$ about $x_{0}$. This neighborhood contains the neighborhood about 0 of radius $r=0.9998|d V(0)|$.

\section{A counter-example for indefinite $g$}

In $[2$, Section 6$]$, it was shown that there exist $u_{h}$ for which

$$
\left\|-h^{2}\left(\partial_{x_{1}}^{2}-\partial_{x_{2}}^{2}\right) u_{h}+\left(x_{1}^{2}-x_{2}^{2}\right) u_{h}\right\|_{L^{2}} \leq h, \quad\left\|u_{h}\right\|_{L^{2}} \leq 1,
$$

for which $\left\|u_{h}\right\|_{L^{\infty}} \approx|\log h|^{\frac{1}{2}} h^{-\frac{1}{2}}$, showing that the assumption of definiteness of $\mathrm{g}$ cannot be relaxed to non-degeneracy in the main theorem. In [2, Theorem 6] the positive result was established showing that this growth of $\left\|u_{h}\right\|_{L^{\infty}}$ for indefinite, non-degenerate $\mathrm{g}$ in two dimensions is in fact worst case.

The example of [2] was produced using harmonic oscillator eigenstates. Here we present a different construction of such a $u_{h}$ with similar $L^{\infty}$ growth to help illustrate the role played by the degeneracy of $\mathrm{g}$. The idea is to produce a collection $u_{h, j}$ of functions satisfying (3.1) (or equivalent), for which $u_{h, j}(0)=h^{-\frac{1}{2}}$, and where $j$ runs over $\approx|\log h|$ different values. The examples will have disjoint frequency support, hence are orthogonal in $L^{2}$. Upon summation over $j$ the $L^{2}$ norm then grows as $|\log h|^{\frac{1}{2}}$, whereas the $L^{\infty}$ norm grows as $|\log h| h^{-\frac{1}{2}}$, yielding an example with worst case growth after normalization.

We start by considering the form $\xi_{1} \xi_{2}$ with $V=0$. To have $\left\|h^{2} \partial_{x_{1}} \partial_{x_{2}} u_{h}\right\|_{L^{2}} \leq h$, we will take the Fourier transform of $u_{h}$ to be contained in the set $\left|\xi_{1} \xi_{2}\right| \leq 2 h^{-1}$, as well as $|\xi| \leq 2 h^{-1}$ to satisfy the frequency localization condition [2, (1.4)]. Our example is then based on the fact that one can find $\approx|\log h|$ disjoint rectangles, each of volume $h^{-1}$, within this region, as illustrated in the diagram. Each $u_{h, j}$ will be an 
appropriately scaled Schwartz function with Fourier transform localized to one of the rectangles.

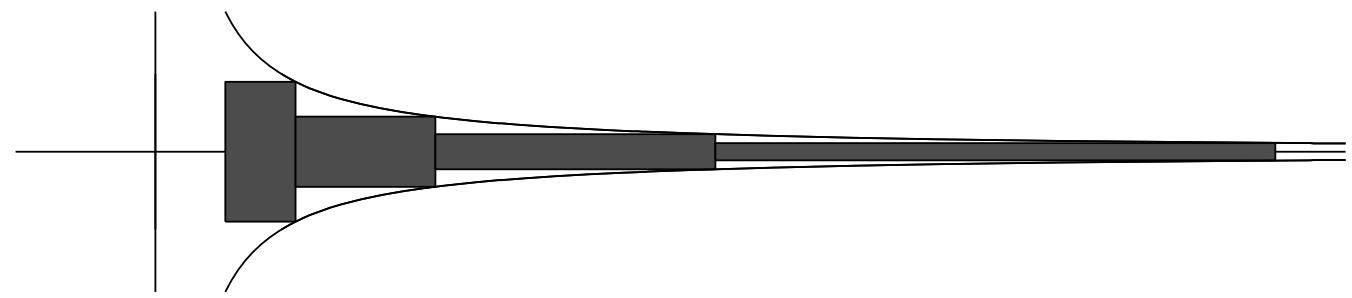

We now fix $\psi, \chi \in \mathcal{C}_{c}^{\infty}(\mathbb{R})$, with $0 \leq \psi(x) \leq 2$ and $0 \leq \chi(x) \leq 1$, with $\int \psi=\int \chi=$ 1 , and where

$$
\operatorname{supp} \psi \subset[1,2], \quad \operatorname{supp} \chi \subset[-1,1]
$$

We additionally assume $\chi(0)=1$.

Let

$$
u_{h, j}(x)=h^{\frac{1}{2}} \int e^{i x_{1} \xi_{1}+i x_{2} \xi_{2}} \chi\left(2^{j} h \xi_{1}\right) \psi\left(2^{-j} \xi_{2}\right) d \xi_{1} d \xi_{2}=h^{-\frac{1}{2}} \check{\chi}\left(2^{-j} h^{-1} x_{1}\right) \check{\psi}\left(2^{j} x_{2}\right) .
$$

By the Plancherel theorem, $\left\|u_{h, j}\right\|_{L^{2}} \approx 1$ and $\left\|h^{2} D_{1} D_{2} u_{h, j}\right\|_{L^{2}} \lesssim h$. Furthermore, $u_{h, j}(0)=h^{-\frac{1}{2}}$. By disjointness of the Fourier transforms, we have $\left\langle u_{h, i}, u_{h, j}\right\rangle=0$ for $i \neq j$, and similarly $\left\langle\partial_{x_{1}} \partial_{x_{2}} u_{h, i}, \partial_{x_{1}} \partial_{x_{2}} u_{h, j}\right\rangle=0$.

We then form

$$
u_{h}(x)=|\log h|^{-\frac{1}{2}} \sum_{1 \leq 2^{j} \leq h^{-1}} u_{h, j}(x) .
$$

Since there are $\approx|\log h|$ terms in the sum, and the terms are orthogonal in $L^{2}$, it follows that

$$
\left\|u_{h}\right\|_{L^{2}} \approx 1, \quad\left\|h^{2} \partial_{x_{1}} \partial_{x_{2}} u_{h}\right\|_{L^{2}\left(\mathbb{R}^{2}\right)} \lesssim h, \quad u_{h}(0) \approx|\log h|^{\frac{1}{2}} h^{-\frac{1}{2}}
$$

Although the example is not compactly supported, it is rapidly decreasing (uniformly so for $h<1$ ), and one may smoothly cutoff to a bounded set without changing the estimates.

We observe that for this example it also holds that

$$
\left\|x_{1} x_{2} u_{h}\right\|_{L^{2}} \lesssim h
$$

Hence, $u_{h}$ is also a counterexample for the form $\xi_{1} \xi_{2} \pm x_{1} x_{2}$. Rotating by $\pi / 4$ gives the form $\xi_{1}^{2}-\xi_{2}^{2} \pm\left(x_{1}^{2}-x_{2}^{2}\right)$, including in particular the form considered in [2, Section $6]$.

We also observe that $x_{1}^{2} u_{h}$ will be $\mathcal{O}_{L^{2}}(h)$ if one restricts the sum in $u_{h}$ to $1 \leq 2^{j} \leq$ $h^{-\frac{1}{2}}$, which still has $\approx|\log h|$ values of $j$, and thus exhibits the same $L^{\infty}$ growth as $u_{h}$. This idea does not however work to yield a counterexample for the form $\xi_{1} \xi_{2}+x_{1}^{2}+x_{2}^{2}$.

\section{Acknowledgments}

This material is based upon work supported by the National Science Foundation under Grants DMS-1161283 and DMS-1201417. 


\section{References}

[1] M. Dimassi and J. Sjöstrand, Spectral Asymptotics in the Semiclassical Limit, Cambridge University Press, 1999

[2] H. Koch, D. Tataru and M. Zworski, Semiclassical $L^{p}$ estimates, Ann. Henri Poincaré 8(5) (2007), 885-916.

[3] C. Sogge, Fourier Integrals in Classical Analysis, Cambridge Tracts in Mathematics 105, Cambridge University Press, 1993.

[4] M. Zworski, Semiclassical Analysis, Graduate Studies in Mathematics 138, AMS, 2012.

Department of Mathematics, University of Washington, Seattle, WA 98195, USA

E-mail address: hart@math.washington.edu

Department of Mathematics, University of California, Berkeley, CA 94720, USA

E-mail address: zworski@math.berkeley.edu 